\title{
Increased RUNX1 expression in patients with immune thrombocytopenia
}

Running title: Increased RUNX1 expression in ITP

Xiaomin Zhong, ${ }^{1}$ Yulu Wu, ${ }^{3}$ Yun Liu, ${ }^{3}$ Feng Zhu, ${ }^{4}$ Xiaoqian Li, ${ }^{3}$ Depeng Li, ${ }^{4}$ Zhenyu Li ${ }^{4}$ Lingyu Zeng, ${ }^{3,4}$ Jianlin Qiao, ${ }^{3,4}$ Xiaofei Chen,${ }^{1 *}$ Kailin $\mathrm{Xu},{ }^{2,3,4^{*}}$

${ }^{1}$ Department of Medical Oncology, Huai'an First People's Hospital, Nanjing Medical University, Huai'an 223300, China

${ }^{2}$ The First Clinical Medical College, Nanjing Medical University, Nanjing 210029, China

${ }^{3}$ Blood Diseases Institute, Xuzhou Medical College, Xuzhou 221002, China

${ }^{4}$ Department of Hematology, the Affiliated Hospital of Xuzhou Medical College, Xuzhou 221002, China

These authors contributed equally to this study as * corresponding author

Address for correspondence:

Kailin Xu, Nanjing Medical University, 140 Hanzhong Rd, Gulou District, Nanjing 210029, Jiangsu, China. Tel: 86-516-8580-2382, Fax: 86-516-8580-1527. Email: lihmd@163.com

Xiaofei Chen, Department of Medical Oncology, Huai'an First People's Hospital, Nanjing Medical University, 6 Beijing Road West, Huai'an, 223300, Jiangsu, China. Email: cxf607001@163.com 


\begin{abstract}
Immune thrombocytopenia (ITP) is a heterogeneous autoimmune disease, characterized by dysregulation of cellular immunity. Th17 and associated IL-17 were involved in the pathogenesis of ITP. Runt-related transcription factor 1 (RUNX1), a member of the runt domain-containing family of transcription factors, is required for Th17 differentiation. Whether RUNX1 was involved in the pathogenesis of ITP remains poorly understood. In this study, 30 active ITP patients, 20 ITP in remission and 20 age and gender matched healthy controls were included. Peripheral blood mononuclear cells (PBMCs) were isolated to measure mRNA level of RUNX1 and retinoic acid receptor-related orphan receptor- $\gamma \mathrm{t}(\mathrm{ROR} \gamma \mathrm{t})$ by quantitative real-time PCR and Th17 cells by flow cytometry. Meanwhile, plasma was extracted for measurement of IL-17 level by ELISA. Our results showed a significantly higher expression of RUNX1, ROR $\gamma \mathrm{t}$, Th17 cells and plasma level of IL-17 in active ITP patients than that in healthy controls. No differences of expression of RUNX1, ROR $\gamma \mathrm{t}$ and Th17 cells were observed between remission patients and controls. Furthermore, a significantly positive correlation of RUNX1 with ROR $\gamma$ t was found in active ITP patients. In conclusion, RUNX1 was associated with the pathogenesis of ITP possibly through regulation of Th17 cell differentiation and therapeutically targeting it might be a novel approach in ITP treatment.
\end{abstract}

Key words: Immune thrombocytopenia, Runt-related transcription factor 1, retinoic acid receptor-related orphan receptor- $\gamma \mathrm{t}$, IL-17 


\section{Introduction}

Immune thrombocytopenia is an autoimmune disease, characterized by antiplatelet autoantibody mediated-immune attack on megakaryocytes and/or platelets, leading to impaired platelet production and/or accelerated platelet destruction and subsequent thrombocytopenia (lower platelet count), putting patients on a higher risk of bleeding [1, 2]. The pathophysiology and pathogenesis of ITP is becoming more and more complicated, involving lots of factors. There is increasing evidence demonstrating that the development of ITP is mainly due to autoantibodies opsonizing individual's platelets, resulting in enhanced $\mathrm{Fc}$ receptor $(\mathrm{FcR})$-mediated phagocytosis as well as destruction by macrophages in the reticuloendothelial system in the spleen [3].

Although antiplatelet autoantibodies have been showed to be critical factors in the pathogenesis of ITP, several other abnormalities or dysregulations including cellular immune modulation have also been identified to play an important role in the pathophysiology of ITP [3, 4]. Given the critical role in the regulation of immune response and attack, abnormal function or activity of $\mathrm{T}$ cells have been observed in patients with ITP [5]. Previous studies showed that less apoptotic and more clonal expansion of platelet autoreactive $\mathrm{T}$ cells in ITP patients, leading to an imbalanced secretion of cytokines and subsequent reduced expression of Tregs, which play a critical role in controlsling appropriate immune response and autoreactivity [6, 7]. T helper cells (Th) 1 and Th2 cells, two T cell subsets, are important for autoimmune reactivity and imbalance of Th1/Th2 has been reported to be associated with ITP [5, 8]. However, in recent years, apart from Th1/Th2, another novel subset of Th cells, IL-17 secreting Th- 
cells (Th17) and associated cytokines (IL-17) are also identified to be implicated in the pathogenesis of ITP [9-11].

Runt-related transcription factor 1 (RUNX1) is a member of the runt domain-containing family of transcription factors. RUNX proteins are a family of evolutionarily conserved heterodimeric transcription factors that have critical roles during the development of many tissues $[12,13]$. RUNX1, also known as AML1a, was identified to be frequently involved in mutations and translocations associated with acute myeloid leukemia [14]. Targeted depletion studies demonstrated the critical role of RUNX1 in hematopoiesis through regulation of the differentiation of hematopoietic stem cells into mature blood cells [15]. In addition, RUNX1 has been also recognized as one of the most important regulatory factors in T-cell immunity through interaction with multiple master regulators involving the differentiation and function of $\mathrm{T}$ cell subset [16]. Previous study showed the critical role of RUNX1 in T cell development as demonstrated by the requirement of RUNX1 for the active repression of $\mathrm{CD}^{-}$and $\mathrm{CD}^{-}$thymocytes [17]. Furthermore, RUNX1 was also required for effective $\beta$-selection and the positive selection of both $\mathrm{CD}^{+}$and $\mathrm{CD}^{+}$thymocytes [18]. In recent years, RUNX1 has been reported to affect Th17 differentiation through interaction with its master regulator retinoic-acid-receptorrelated orphan receptor- $\gamma \mathrm{t}$ (ROR $\gamma \mathrm{t}$ ) [19]. Meanwhile, RUNX1 is required for IL-17 transcription in Th17 cells by acting cooperatively with ROR $\gamma \mathrm{t}$ [20].

Given the implication of abnormality of Th17 cells in the pathogenesis of ITP and RUNX1 in Th17 cells differentiation, whether RUNX1 is associated with ITP remains unclear. In this study, we evaluate the expression profile of RUNX1 in patients with active ITP as well as ITP patients with remission. 


\section{Materials and Methods}

\subsection{Patients}

30 primary ITP patients (10 males and 20 females with a median age of 40 , ranged from 18 to 75 years old) (Table 1) from Department of Hematology, the Affiliated Hospital of Xuzhou Medical College, Xuzhou China were enrolled in this study. The diagnosis of ITP (newly diagnosed, persistent and chronic) was based on the criteria from international working group [21, 22]. These primary ITP patients with a platelet count < $50 \times 10^{9} / \mathrm{L}$ were defined as active ITP (clinical characteristics in supplementary Table S1), who had a median platelet count of $20 \times 10^{9} / \mathrm{L}$, ranged from 6 to $45 \times 10^{9} / \mathrm{L}$. No patients had a secondary cytopenia like Evans Syndrome or another autoimmune disease. Patients with hypertension, cardiovascular diseases, diabetes, active or chronic infection were excluded. Meanwhile, 20 ITP patients ( 7 males and 13 females with a median age of 42 ranged from 19 to 80 years old) (Table 1) who were in remission after treated with glucocorticoids or IVIG with a median course of disease being 10 months (ranging from 0-51) were also included in this study and their platelet counts ranged from 100 to $261 \mathrm{x}$ $10^{9} / \mathrm{L}$ with a median count of $186 \times 10^{9} / \mathrm{L} .20$ age and gender-matched healthy individuals (6 males and 14 females with a median age of 43, ranged from 23 to 79 years old) (Table 1) were served as a controls, whose platelet counts ranged from 163 to $305 \mathrm{x}$ $10^{9} / \mathrm{L}$ with a median count of $225 \times 10^{9} / \mathrm{L}$. Ethical approval for this study was obtained from the Medical Ethics Committee of the Affiliated Hospital of Xuzhou Medical College, Xuzhou China. This study has been carried out in accordance with The Code of Ethics of the World Medical Association (Declaration of Helsinki) for experiments 
involving humans. Informed consent was obtained from all participants before enrollment in the study.

\subsection{Plasma isolation}

Venous whole blood was drawn from patients or controls followed by transferring into tubes with EDTA as anti-coagulant. Plasma was acquired through centrifuging anticoagulant blood at $2000 \mathrm{rpm}$ for $20 \mathrm{~min}$ and stored at $-80^{\circ} \mathrm{C}$ until further analysis of IL-17 level by ELISA.

\subsection{Isolation of peripheral blood mononuclear cells and RNA extraction}

Peripheral blood mononuclear cells (PBMCs) were isolated from venous whole blood by gradient centrifugation on Ficoll-Paque Plus (Sinopharm Chemical Reagent Ltd, China) at $2000 \mathrm{rpm}$ for $20 \mathrm{~min}$. RNA was extracted from isolated PBMCs by using TRIZOL reagent (Life Technology, Carlsbad, CA, USA) according to manufacturer's instruction. RNA quantitation and quality was assessed by measuring the absorbance of RNA at 260 $\mathrm{nm}$ on a spectrophotometer and agarose gels, respectively.

\subsection{Real-time quantitative PCR (RT-qPCR)}

RT-qPCR was performed as previously described [23, 24]. Extracted RNA was reversedtranscripted to cDNA which was used for quantification of mRNA expression of RUNX1 and ROR $\gamma$ t by real-time PCR with GADPH as internal controls. Amplification was

performed in triplicate on LightCycler ${ }^{\circledR} \mathrm{R} 480$ II (Roche Life Science) in a total volume of $20 \mu \mathrm{l}$, including $10 \mu \mathrm{l}$ SYBR Green qPCR Super Mix, $0.5 \mu \mathrm{l}$ forward primer $(10 \mu \mathrm{M}), 0.5$ $\mu \mathrm{l}$ reverse primer $(10 \mu \mathrm{M}), 5 \mu \mathrm{l} \mathrm{cDNA}$ and $4 \mu \mathrm{l}$ sterile water. The primers for RUNX3, ROR $\gamma$ t and GADPH were shown in Table 2. The PCR reaction conditions were as 
follows: Initial denaturation $\left(95^{\circ} \mathrm{C}\right.$ for $\left.5 \mathrm{~min}\right), 40$ cycles of denaturation $\left(95^{\circ} \mathrm{C}\right.$ for $\left.20 \mathrm{~s}\right)$; annealing $\left(60^{\circ} \mathrm{C}\right.$ for $\left.15 \mathrm{~s}\right)$ and extension $\left(72^{\circ} \mathrm{C}\right.$ for $\left.15 \mathrm{~s}\right)$. The melting curve analysis of the amplification products was performed at the end of each PCR reaction. The relative mRNA expression of target genes was calculated by comparative Ct method which is performed by using the following formula: relative expression $=2^{-\Delta \Lambda} \mathrm{Ct}$.

\subsection{Flow cytometry}

Isolated PBMCs were resuspended in RPMI-1640 medium supplemented with $100 \mathrm{U} / \mathrm{ml}$ penicillin, $100 \mu \mathrm{g} / \mathrm{ml}$ streptomycin and $10 \%$ heat-inactivated fetal calf serum (Gibco BRL) at a density of $1 \times 10^{6}$ cells $/ \mathrm{ml}$ and then transferred to each well of 24-well plate. The cultured cells were stimulated with PMA (50 ng/ml) (Sigma-Aldrich, St. Louis, MO, USA), ionomycin (750 ng/ml) (Sigma-Aldrich) and BFA (10 $\mu \mathrm{g} / \mathrm{ml})$ (Invitrogen, Carlsbad, CA, USA) followed by moving the plates into a $37^{\circ} \mathrm{C}$ incubator with $5 \% \mathrm{CO}_{2}$ for 4 hours. At the end, the cells were transferred into sterile tubes and centrifuged at 350 $\mathrm{g}$ for 5 min. After that, FITC-conjugated anti-human CD4 antibody (eBioscience, San Diego, CA, USA) was added into cells and incubated for $15 \mathrm{~min}$ at room temperature under dark. Then, the cells were fixed and centrifuged followed by permeabilization and addition of APC-conjugated anti-human IL-17A (eBioscience) for 30 min and analyzed in a FACSCalibur (Becton Dickinson, San Jose, CA, USA). Corresponding isotype antibodies (eBioscience) were used as a control. CD4+ IL-17+ cells were defined as Th17 cells. 


\subsection{Statistical analysis}

Data was presented as Mean \pm SE. Using GraphPad Prism software, one-way ANOVA was used for the comparison of relevant parameters among different groups. For correlation, liner regression analysis was performed. $\mathrm{P}$ value less than 0.05 was considered to be statistically significant.

\section{Results}

\subsection{Expression of RUNX1 in ITP patients and controls}

Peripheral blood mononuclear cells were isolated from active ITP patients, ITP patients in remission or healthy controls for measurement of the expression level of RUNX1 by RT-qPCR. As seen in Figure 1, a significantly higher expression level of RUNX1 was observed in active ITP patients $(6.55 \pm 1.65)$ compared to controls $(0.71 \pm 0.14)(\mathrm{P}<$ $0.01)$. RUNX1 expression was dramatically reduced in patients in remission $(0.84 \pm 0.12)$ than that in active ITP patients $(\mathrm{P}<0.01)$ with no significant difference compared with controls $(\mathrm{P}>0.01)$.

\subsection{Expression of ROR 2 and Th17 cells in ITP patients and controls}

Given the critical role of RUNX1 in the regulation of Th17 cell differentiation through interacting with its master regulator ROR $\gamma t[19,20]$, the expression level ROR $\gamma t$ in ITP patients and healthy controls was also measured by RT-qPCR. As seen in Figure 2, ROR $\gamma$ t expression was significantly higher in active ITP patients $(05.02 \pm 1.03)$ than that in healthy controls $(0.99 \pm 0.22)(\mathrm{P}<0.01)$, suggesting the abnormal Th17 cells in ITP, consistent with the previously studies showing the association of Th17 differentiation 
with the pathogenesis of ITP [9-11]. However, its level was significantly lower in patients in remission $(1.37 \pm 0.40)$ than that in active patients $(\mathrm{P}<0.01)$ without significant difference to controls $(\mathrm{P}>0.05)$. Consistent with changes of ROR $\gamma$ t, levels of Th17 cells were significantly higher in active ITP patients than controls $(\mathrm{P}<0.05)$, but restored into normal levels in patients in remission $(\mathrm{P}>0.05)$. Taken together, these data further supported the role of Th17 cells in the development of ITP.

\subsection{Correlative analysis of RUNX1 with ROR $\gamma t$}

In light of the similar expression patterns of RUNX1 and ROR $\gamma$ t in active ITP patients and ITP patients in remission, a correlative analysis was performed to evaluate their relationship in the pathogenesis of ITP. As seen in Figure 3, a significant positive correlation between RUNX1 and ROR $\gamma \mathrm{t}$ was observed in active ITP patients $(\mathrm{R}=0.569$, $P=0.002$ ), suggesting an interrelationship between RUNX1 and ROR $\gamma t$ might contribute to Th17 cell differentiation in the development of ITP, consistent with previous studies demonstrating the requirement of the interaction between RUNX1 and ROR $\gamma t$ during Th17 differentiation $[19,20]$.

\subsection{IL-17 plasma level}

Through cooperatively interacting with ROR $\gamma t$, RUNX1 was demonstrated to be required for the transcription of IL-17 in Th17 cells [20]. To evaluate whether dysregulated RUNX1 and ROR $\gamma$ t affect IL-17 production in ITP patients, IL-17 level was measured in the plasma from ITP patients or controls. Consistent with expression profile of RUNX1 and ROR $\gamma$ t, IL-17 plasma level was significantly higher in active ITP patients $(5.75 \pm$ $0.37 \mathrm{ng} / \mathrm{l})$ than that in controls $(2.73 \pm 0.44 \mathrm{ng} / \mathrm{l})(\mathrm{P}<0.01)$. However, IL-17 level was 
dramatically decreased in ITP patients in remission $(4.33 \pm 0.46)$ compared to active ITP patients $(5.75 \pm 0.37 \mathrm{ng} / \mathrm{l})(\mathrm{P}<0.05)$. Even with a reduced IL-17 level in remission patients $(4.33 \pm 0.46 \mathrm{ng} / \mathrm{l})$, there was still significantly higher than controls $(2.73 \pm 0.44$ ng/l) $(\mathrm{P}<0.05)$. Compared to no difference of expression of RUNX1 or ROR $\gamma t$ between controls and remission patients, difference of IL-17 level between controls and remission patients suggested IL-17 might be originated from other cells apart from Th17 cells.

\section{Discussion}

Immune thrombocytopenia is a heterogeneous autoimmunity disease, characterized by immune cells-mediated platelet destruction and/or platelet production defect, leading to a lower platelet count, putting patients on a higher risk of bleeding [1]. More and more evidence demonstrated that imbalance of the differentiation of Th1 and Th2 cells play an important role in the development and pathogenesis of ITP [5]. However, in recent years, abnormal Th17 cells differentiation was reported to be another factor contributing to the development of ITP [25]. Given the critical role in the regulation of the differentiation of Th17 cells, whether RUNX1 was involved in ITP remains poorly understood. In the present study, we demonstrated abnormal expression of RUNX1 and ROR $\gamma \mathrm{t}$ (a master regulator of Th17 cell differentiation) were observed in active ITP patients and restored into normal values in patients in remission.

Runt-related transcription factor (RUNX) proteins are a family of heterodimeric transcription factors, characterized by the presence of a Runt domain, a highly conserved 128-amino acid region [26]. The RUNX family is composed of three members, RUNX1, 
RUNX2 and RUNX3, each of which forms a functional complex with a core binding factor $\beta(\operatorname{Cbf} \beta)$ partner protein. Through mouse genetic studies, a major aspect of RUNX's contribution to immune function has been identified to be involved in the differentiation and maturation of T cells [27]. The RUNX1 protein is highly expressed in $\mathrm{T}$ lymphocytes and has been shown to regulate a diverse aspect of $\mathrm{T}$ cell differentiation, ranging from the early development of thymocyte to the peripheral differentiation of Th cells [16]. During early development of T lymphocyte, RUNX1 orchestrates T-cell receptor (TCR) rearrangement events through binding to the corresponding TCR chain enhancers [28]as well as inhibits CD4 expression, leading to promoted differentiation of CD4/CD8 double negative cells [18]. In addition, RUNX1 also plays an important role in the positive selection of CD4 single positive cells [18]. During T cell differentiation, overexpression of RUNX1 promotes the differentiation of CD4+ T cells into Th1 cells through repression of GATA3 expression, a master regulator of Th2 cell differentiation [16]. In the present study, we demonstrated a significantly higher expression of RUNX1 in active ITP patients compared to controls, which was restored into normal value in ITP patients in remission, suggesting association of abnormal expression of RUNX1 with the development of ITP. However, a recent study conducted by Bal et al., demonstrated decreased RUNX1 expression in chronic ITP patients [29]. The difference to our study might be the clinical characteristics of ITP patients, who are classified as being in active phase or remission phase in Bal's study and active ITP patients in our study. In addition, different treatments might also be accounted for this discrepancy.

Previous studies demonstrated that RUNX transcription factors regulate target gene expression in a context-dependent manner via interactions with other transcription factors 
to activate or repress gene expression [30]. RUNX proteins have been shown to regulate appropriate Th cell differentiation and cytokine production through forming a complex with other transcription factors [20]. Our previous study showed that interaction of RUNX3 with T-bet enhances Th1 cell differentiation through up-regulation of IFN- $\gamma$ expression in patients with ITP [31]. In addition, by promotion ROR $\gamma$ t expression, RUNX1 appears to favor Th17 phenotype through forming a complex with ROR $\gamma \mathrm{t}$ [20]. Similar to the expression profile of RUNX1, a significantly higher expression of ROR $\gamma t$ was observed in active ITP patients compared to controls. However, this expression was reduced in patients in remission without difference to controls. These data suggested that elevated expression of ROR $\gamma \mathrm{t}$ might be resulted from abnormal RUNX1 expression, further supported by a significantly positive correlation of RUNX1 with ROR $\gamma \mathrm{t}$ in active ITP patients, indicating that cooperative interaction between RUNX1 and ROR $\gamma \mathrm{t}$ might contribute to the pathogenesis of ITP.

Th cells, differentiated from naïve $\mathrm{CD} 4+\mathrm{T}$ cells upon stimulation by antigen in the presence of different cytokines produced by immune cells, are demonstrated to play important role in the regulation of immune system, particular in adaptive immune response [32]. Th1 and Th2 cells are two major Th cell subsets, characterized by secretion of IFN- $\gamma$ and IL-4 respectively [32]. Later on, another subset of helper CD4+ T cells, Th17 lineage was identified, which produces IL-17 as a signature cytokine [33]. As a pro-inflammatory cytokine, IL-17 plays an essential role in host defense against microbial infections and is implicated in multiple inflammatory conditions such as autoimmune diseases, metabolic disorders, and cancer [34]. The main function of IL-17 is to induce the production of cytokines, chemokines, acute phase proteins and anti- 
microbial peptides, leading to recruitment of neutrophils and monocytes at the site of $\mathrm{T}$ cell activation [35]. Consistent with elevated Th17 cells as demonstrated by higher expression of ROR $\gamma$ t in this study, we also showed that IL-17 level was a significantly higher in active ITP patients than that in healthy controls. However, even reduced in remission patients, IL-17 level was still significantly higher than controls, suggesting IL17 might be originated from other cells rather than Th17 cells only, consistent with previous studies revealing IL-17 can also be produced by other cell types, such as CD8+ T cells, lymphoid tissue inducer cells, NK cells and macrophages.

In conclusion, our study demonstrated a significantly higher expression of RUNX1 and ROR $\gamma$ t in active ITP patients, which was restored into normal value in remission patients. Meanwhile, a positively correlation of RUNX1 with ROR $\gamma \mathrm{t}$ was found in active patients. Our results indicated that RUNX1 might be involved in the pathogenesis of ITP possibly through regulation of Th17 cell differentiation and targeting it may be a new therapeutic approach in the treatment of ITP.

\section{Acknowledgements}

This research was supported by National Science Foundation of China (81270637).

\section{Conflict of Interest}

The authors have no conflict of interest to declare. 


\section{References}

[1] Cines DB, Cuker A, Semple JW: Pathogenesis of immune thrombocytopenia. Presse Med 2014;43:e49.

[2] Cines DB, Bussel JB, Liebman HA, Luning Prak ET: The ITP syndrome: pathogenic and clinical diversity. Blood 2009;113:6511.

[3] McKenzie CG, Guo L, Freedman J, Semple JW: Cellular immune dysfunction in immune thrombocytopenia (ITP). Br J Haematol 2013;163:10.

[4] Liu X, Hou Y, Peng J: Advances in immunopathogenesis of adult immune thrombocytopenia. Front Med 2013;7:418.

[5] Ji X, Zhang L, Peng J, Hou M: T cell immune abnormalities in immune thrombocytopenia. J Hematol Oncol 2014;7:72.

[6] Ma L, Zhou Z, Zhang D, Wang H, Li H, Xue Fet al. : Bmi-1 regulates autoreactive CD4+ T cell survival in immune thrombocytopenia patients. J Clin Immunol 2012;32:505.

[7] Yu J, Heck S, Patel V, Levan J, Yu Y, Bussel JBet al. : Defective circulating CD25 regulatory $\mathrm{T}$ cells in patients with chronic immune thrombocytopenic purpura. Blood 2008;112:1325.

[8] Wang T, Zhao H, Ren H, Guo J, Xu M, Yang Ret al. : Type 1 and type 2 T-cell profiles in idiopathic thrombocytopenic purpura. Haematologica 2005;90:914.

[9] Hu Y, Li H, Zhang L, Shan B, Xu X, Liu Xet al. : Elevated profiles of Th22 cells and correlations with Th17 cells in patients with immune thrombocytopenia. Hum Immunol 2012;73:629. 
[10] Ji L, Zhan Y, Hua F, Li F, Zou S, Wang Wet al. : The ratio of Treg/Th17 cells correlates with the disease activity of primary immune thrombocytopenia. PLoS One 2012;7:e50909.

[11] Semple JW, Provan D: The immunopathogenesis of immune thrombocytopenia: T cells still take center-stage. Curr Opin Hematol 2012;19:357.

[12] Ito Y: RUNX genes in development and cancer: regulation of viral gene expression and the discovery of RUNX family genes. Adv Cancer Res 2008;99:33.

[13] Speck NA: Core binding factor and its role in normal hematopoietic development. Curr Opin Hematol 2001;8:192.

[14] Yamagata T, Maki K, Mitani K: Runx1/AML1 in normal and abnormal hematopoiesis. Int J Hematol 2005;82:1.

[15] Okuda T, van Deursen J, Hiebert SW, Grosveld G, Downing JR: AML1, the target of multiple chromosomal translocations in human leukemia, is essential for normal fetal liver hematopoiesis. Cell 1996;84:321.

[16] Wong WF, Kohu K, Chiba T, Sato T, Satake M: Interplay of transcription factors in T-cell differentiation and function: the role of Runx. Immunology 2011;132:157.

[17] Woolf E, Xiao C, Fainaru O, Lotem J, Rosen D, Negreanu Vet al. : Runx3 and Runx1 are required for CD8 T cell development during thymopoiesis. Proc Natl Acad Sci U S A 2003;100:7731.

[18] Egawa T, Tillman RE, Naoe Y, Taniuchi I, Littman DR: The role of the Runx transcription factors in thymocyte differentiation and in homeostasis of naive $\mathrm{T}$ cells. J Exp Med 2007;204:1945. 
[19] Ivanov, II, McKenzie BS, Zhou L, Tadokoro CE, Lepelley A, Lafaille JJet al. : The orphan nuclear receptor RORgammat directs the differentiation program of proinflammatory IL-17+ T helper cells. Cell 2006;126:1121.

[20] Zhang F, Meng G, Strober W: Interactions among the transcription factors Runx1, RORgammat and Foxp3 regulate the differentiation of interleukin 17-producing $\mathrm{T}$ cells. Nat Immunol 2008;9:1297.

[21] Rodeghiero F, Stasi R, Gernsheimer T, Michel M, Provan D, Arnold DMet al. : Standardization of terminology, definitions and outcome criteria in immune thrombocytopenic purpura of adults and children: report from an international working group. Blood 2009;113:2386.

[22] Provan D, Stasi R, Newland AC, Blanchette VS, Bolton-Maggs P, Bussel JBet al. : International consensus report on the investigation and management of primary immune thrombocytopenia. Blood 2010;115:168.

[23] Qiao J, Liu Y, Li X, Xia Y, Wu Y, Li Det al. : Elevated expression of NLRP3 in patients with immune thrombocytopenia. Immunol Res 2015.

[24] Qiao J, Liu Y, Li D, Wu Y, Li X, Yao Yet al. : Imbalanced expression of Bcl-xL and Bax in platelets treated with plasma from immune thrombocytopenia. Immunol Res 2015.

[25] Zhang J, Ma D, Zhu X, Qu X, Ji C, Hou M: Elevated profile of Th17, Th1 and Tc1 cells in patients with immune thrombocytopenic purpura. Haematologica 2009;94:1326. 
[26] Kagoshima H, Shigesada K, Satake M, Ito Y, Miyoshi H, Ohki Met al. : The Runt domain identifies a new family of heteromeric transcriptional regulators. Trends Genet 1993;9:338.

[27] Collins A, Littman DR, Taniuchi I: RUNX proteins in transcription factor networks that regulate T-cell lineage choice. Nat Rev Immunol 2009;9:106.

[28] Cieslak A, Le Noir S, Trinquand A, Lhermitte L, Franchini DM, Villarese Pet al. : RUNX1-dependent RAG1 deposition instigates human TCR-delta locus rearrangement. J Exp Med 2014;211:1821.

[29] Bal G, Futschik ME, Hartl D, Ringel F, Kamhieh-Milz J, Sterzer Vet al. : Identification of novel biomarkers in chronic immune thrombocytopenia (ITP) by microarray-based serum protein profiling. Br J Haematol 2016;172:602.

[30] Mikhail FM, Sinha KK, Saunthararajah Y, Nucifora G: Normal and transforming functions of RUNX1: a perspective. J Cell Physiol 2006;207:582.

[31] Qiao J, Liu Y, Wu Y, Li X, Zhu F, Xia Yet al. : Aberrant expression of RUNX3 in patients with immune thrombocytopenia. Int Immunopharmacol 2015;28:252.

[32] Luckheeram RV, Zhou R, Verma AD, Xia B: CD4(+)T cells: differentiation and functions. Clin Dev Immunol 2012;2012:925135.

[33] McGeachy MJ, Cua DJ: Th17 cell differentiation: the long and winding road. Immunity 2008;28:445.

[34] Gu C, Wu L, Li X: IL-17 family: cytokines, receptors and signaling. Cytokine $2013 ; 64: 477$

[35] Isailovic N, Daigo K, Mantovani A, Selmi C: Interleukin-17 and innate immunity in infections and chronic inflammation. J Autoimmun 2015;60:1. 
Tables

Table 1. Basic information about ITP patients and healthy controls.

\begin{tabular}{cccc}
\hline & Active ITP & ITP in remission & Controls \\
\hline $\mathbf{N}$ & 30 & 20 & 20 \\
Male/Female & $10 / 20$ & $7 / 13$ & $6 / 14$ \\
Age (Median, Range) & $40(18-75)$ & $42(19-80)$ & $43(23-79)$ \\
$\begin{array}{c}\text { Platelet count } \\
(\text { Median, Range) } \\
(\mathbf{x ~ 1 0} / \mathbf{L})\end{array}$ & $20(6-45)$ & $168(100-261)$ & $233(163-305)$ \\
\hline
\end{tabular}


Table 2. Primers for real-time quantitative PCR.

\begin{tabular}{llc}
\hline Gene & Primer Sequence (5'-3') & Length (bp) \\
\hline \multirow{2}{*}{ RUNX1 } & F: TTCACAAACCCACCGCAAGT & 134 \\
& R: CGCTCGGAAAAGGACAAGC & \\
ROR $\boldsymbol{y t}$ & F: CTGCTGAGAAGGACAGGGAG & 202 \\
& R: AGTTCTGCTGACGGGTGC & \\
& F: TGAAGGTCGGAGTCAACGGATT & 225 \\
\hline
\end{tabular}




\section{Figure legends}

Figure 1. mRNA expression of RUNX1 in healthy controls and ITP patients. Peripheral blood mononuclear cells were isolated from healthy controls, active ITP patients or ITP patients in remission. RNA was then isolated for measuring expression of RUNX1 by real-time quantitative PCR.

Figure 2. Expression of ROR $\gamma$ t and Th17 cells in healthy controls and ITP patients.

Peripheral blood mononuclear cells were isolated from healthy controls, active ITP patients or patients in remission followed by extraction of RNA for measuring expression of ROR $\gamma$ t by real-time quantitative PCR (A) and Th17 cells by flow cytometry (B).

Figure 3. Correlative analysis between RUNX1 and ROR $\gamma$ t. Relative mRNA levels of RUNX1 and ROR $\gamma t$ in active ITP patients were measured by real-time quantitative PCR. Correlation between RUNX1 and ROR $\gamma \mathrm{t}$ was analyzed by linear regression.

Figure 4. IL-17 plasma level in healthy controls and ITP. Peripheral whole blood was collected from controls or ITP patients followed by plasma isolation. IL-17 Plasma level was measured by ELISA. 
Figure 1

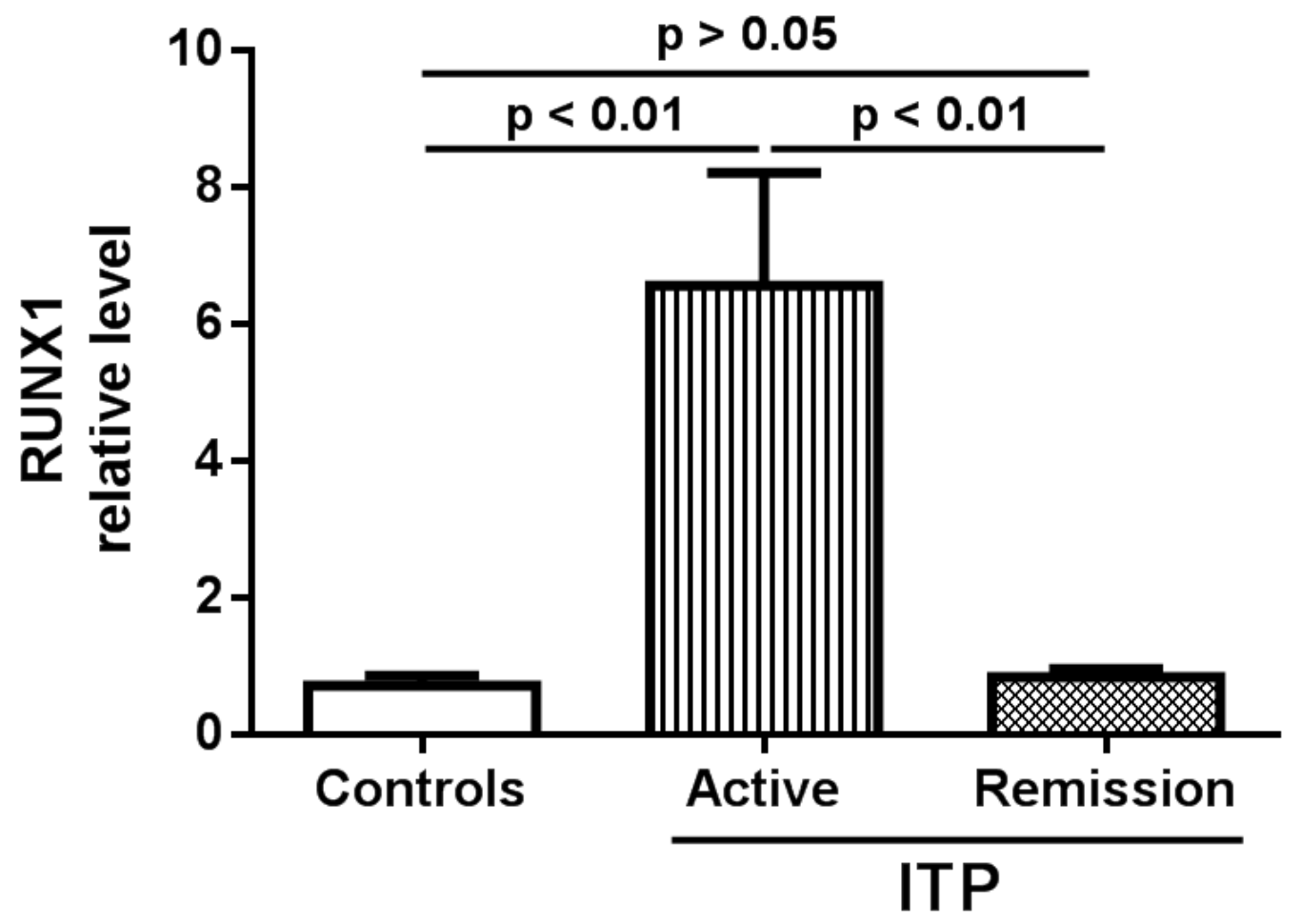


Figure 2
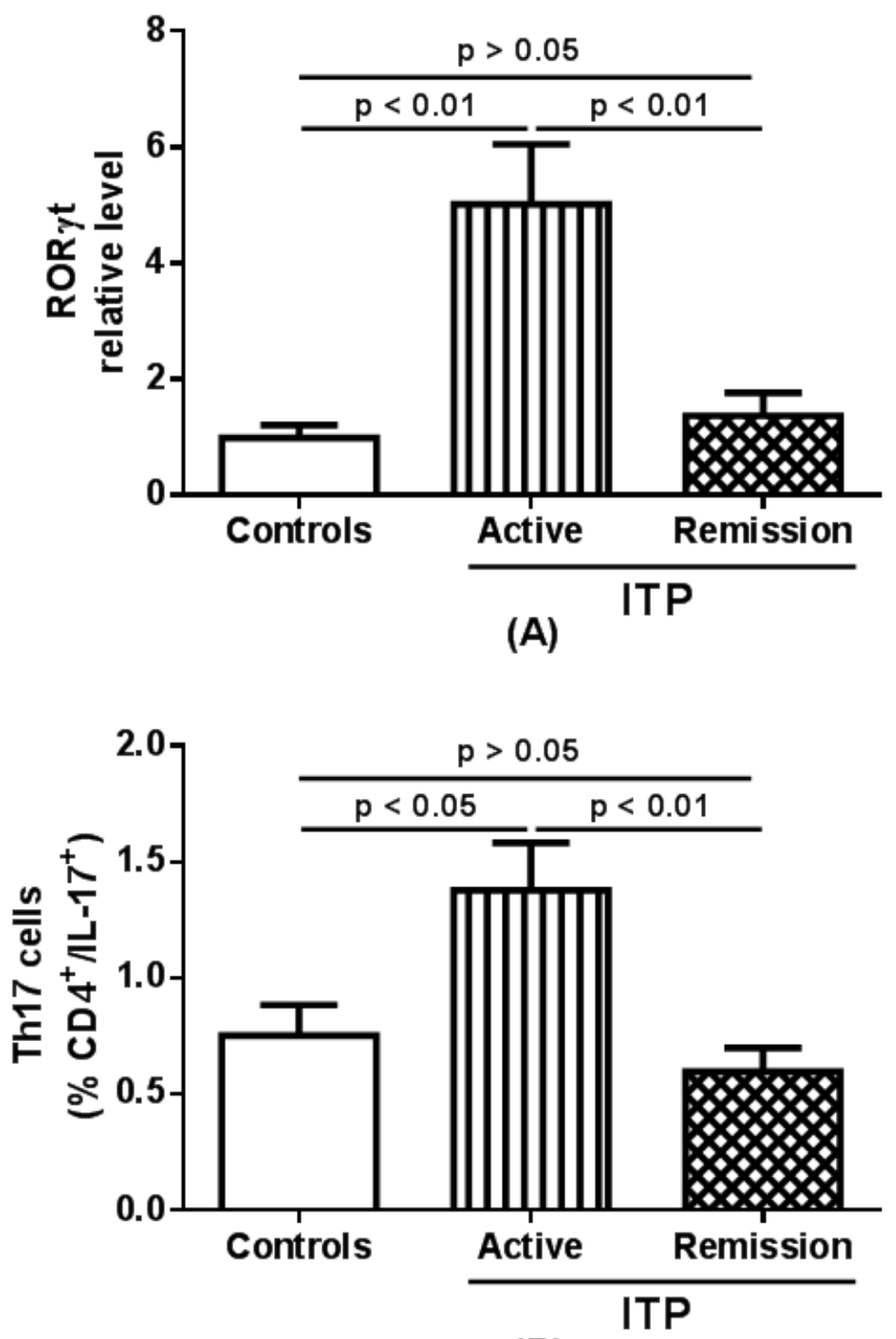

(B) 
Figure 3

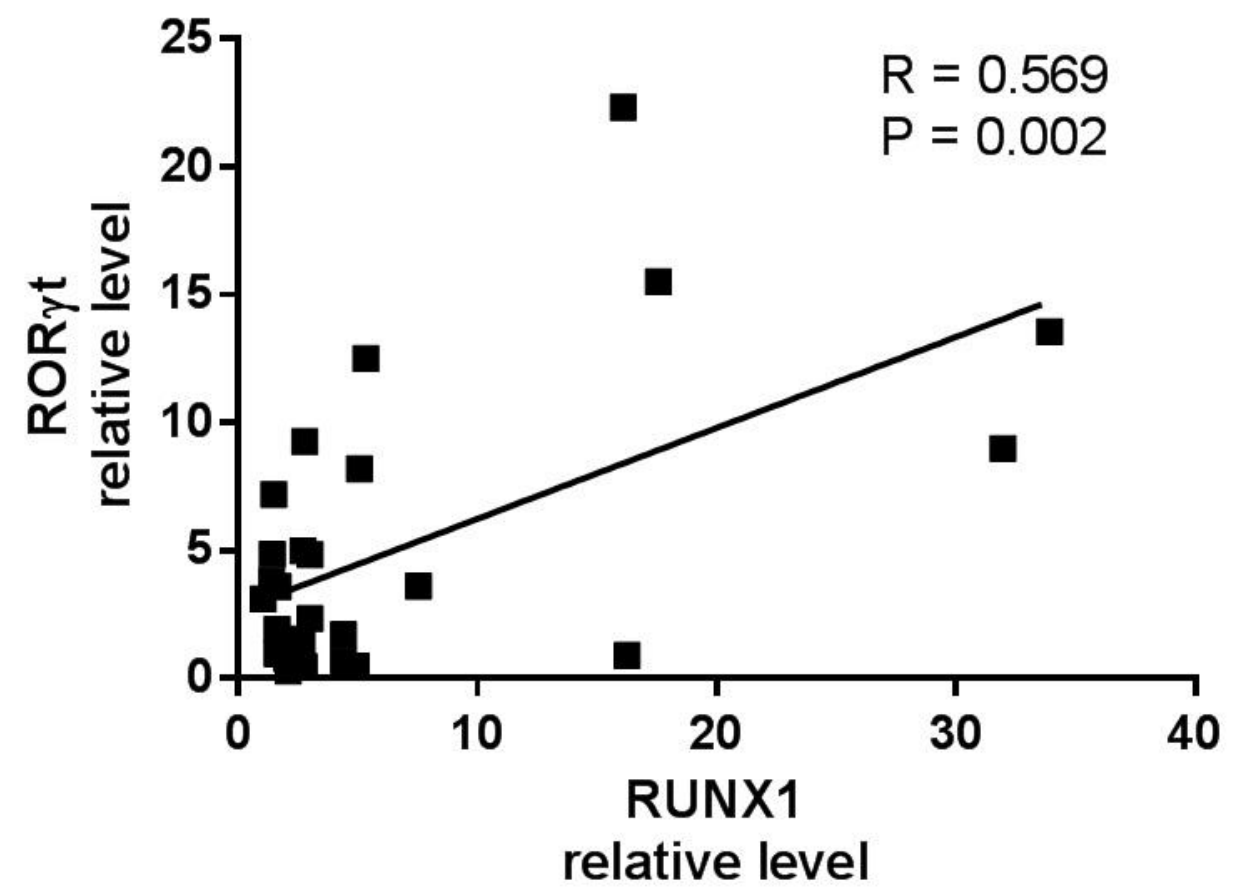


Figure 4

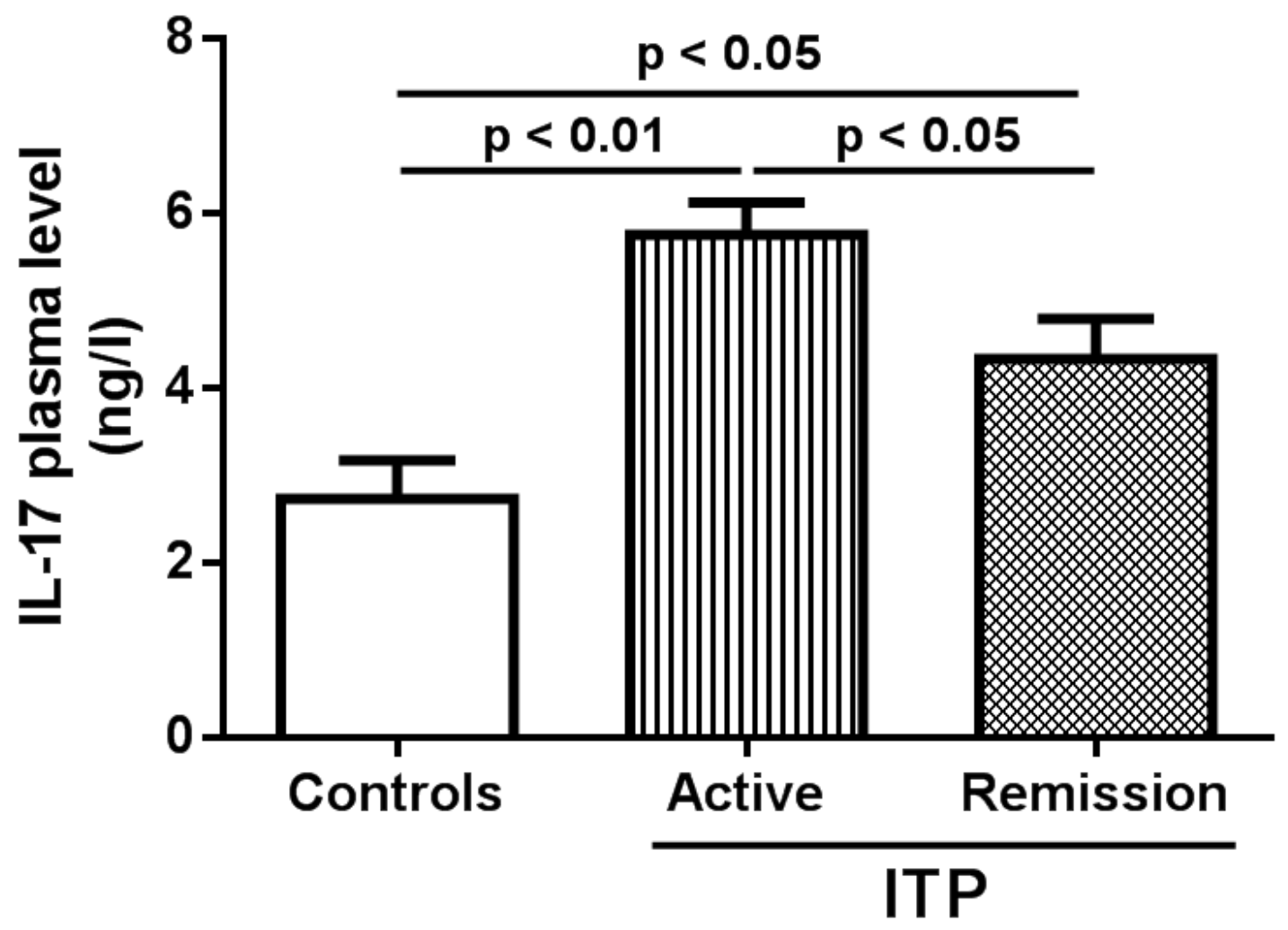

